\title{
Robust stability of time-delay systems with interval delays
}

\author{
Frédéric Gouaisbaut and Dimitri Peaucelle *
}

March 9, 2007

\begin{abstract}
Stability analysis of uncertain time-delay systems is considered in a quadratic separation framework. Results are formulated in terms of linear matrix inequalities (LMI) which prove to be conservative. But this conservatism is significantly reduced by combining several tools: First, a discretization of the delay is performed thus introducing knowledge on the functional defining the current state of the system. Second, a Taylor series approximation of the delay operator is performed and the Taylor remainder is explicitly taken into account with a new uncertainty-type approximation. Thanks to LMI formulation, and with the use of slack variables, robustness is guaranteed for all uncertainties and all delays included in some bounded interval which may not include zero.

Keywords: Linear time-delay systems, Stability, LMIs, Robustness, quadratic separation, Taylor series.
\end{abstract}

\section{Introduction}

Functional differential equations of retarded type with constant delays are often used for modelling practical issues in the engineering field as well as for biology, economics or ecology (see [11] for examples). Stability analysis and stabilization of such systems is therefore an important research topic and several approaches have been successfully proposed. For the important case of linear systems one can focus on three techniques.

The first classical technique relies on the study of the roots of the associated characteristic equation, a quasi-polynomial in $s$, the derivative operator, and $e^{-h s}$, the delay operator. Even if very effective in practice (see $[13,14]$ and the monograph [7]), these techniques are often restricted to nominal stability analysis and cannot extend to robustness issues or design problems.

${ }^{*}$ Frédéric Gouaisbaut and Dimitri Peaucelle are with LAAS-CNRS, University of Toulouse, 7 avenue du Colonel Roche, 31077 Toulouse, FRANCE \{gouaisbaut, peaucelle\} @ laas.fr 
A second popular method, adapted from the non-delayed case, relies on a LyapunovKrasovskii functional. For linear systems, the general structure of the LyapunovKrasovskii functional is provided but is hard to handle in practice. According to the important literature devoted to this subject $[16,19]$, conservative results can still be formulated in terms of linear matrix inequalities (LMIs) if making some restrictions on the Lyapunov-Krasovskii functional.

Yet another important framework is derived from robustness analysis. The core idea is to model the time-delay system as a nominal system connected to an uncertain operator composed as combinations of $s^{-1}$ and $e^{-s h}$. The sources of conservatism are twofold: description of the uncertain operator as member of an uncertainty set and the robustness analysis tools converting the formulated problem into LMIs. This technique is adopted in the current paper and the focus is made on improving uncertainty set description.

An initial simple approach is to describe $e^{-s h}$ as member of the unit disc when $s$ has positive real part and $h$ is positive. Based on such description, independent of delay results are derived. These are conservative due to robustness analysis tools, but conservatism may be reduced asymptotically to zero [2]. An interpretation of the conservatism reduction is given in [15] in terms of artificial model augmentation where the differential equations are further derived thus introducing the knowledge of delay being constant.

A second approach [20,4], proposes to introduce the operator $\left(1-e^{-h s}\right) s^{-1}$ which is member of the circle centered at zero with radius $h$. It thus allows to derive delaydependent results but is limited to the analysis of systems stable for zero delay. Again, the result is conservative due to robustness analysis tools, but conservatism may be reduced by fractioning the delay [4]: $\left(1-e^{-h s / q}\right) s^{-1}$ is bounded in a smaller disc with radius $h / q$. Fractioning does not allow to reduce asymptotically conservatism to zero but may be combined to model augmentation with higher derivatives as done in [6]. Combining fractioning and artificial model augmentation is one of the contributions of the paper.

An alternative to higher order derivatives model augmentation is Padé approximation of $e^{-h s}$ as done in [21,3]. Similarly to model augmentation, it introduces extra dynamic equations corresponding to the transfer function of the Padé approximate operated on all the system states. Of course Padé approximation is not exact, but the gap is modeled as an uncertainty which needs to be properly described, making operator description a key issue.

Although of practical importance in engineering fields like combustion [1], chatter stability in machining [9] and others, only recently stability of time-delay systems which are unstable for zero delay (interval delay stability) has proved to have LMI conservative solutions. One of these is based on the Padé approximate approach [10]. Another one is based on Taylor series approximation [6]. It takes advantage of the system augmentation via derivatives of the state, to explicitly introduce an operator representing the Lagrange remainder. This operator happens to be exactly $e^{-h s}$ for zero degree Taylor series expansion and $\left(1-e^{-h s}\right) s^{-1}$ for first degree expansion. While in [6] all 
the remainders were described in discs centered at zero, the current paper improves the uncertainty description with other discs reduced in size.

Following the ideas developed in [4, 6], quadratic separation techniques (see [8] and extensions in [15]) are used to derive LMI conditions for uncertain models. The models are those obtained while applying both fractioning of the delay and higher order derivatives. The uncertain operators are the Lagrange remainders and are bounded in properly adapted discs. The LMI results are proved to be less and less conservative as the fractioning and the order of derivatives grow.

The paper is organized as follows. Next section is devoted to some preliminaries about quadratic separation. Then section III formulates precisely the Taylor series and fractioning schemes. The following section gives the central results of the paper and discusses conservatism reduction. Section $\mathrm{V}$ extends the results to robust stability with interval delays.

\section{Preliminaries}

\subsection{Notations}

For a two symmetric matrices, $A$ and $B, A>(\geq) B$ means that $A-B$ is (semi-) positive definite. $A^{T}$ denotes the transpose of $A . \mathbf{1}_{n}$ and $\mathbf{0}_{m, n}$ denote the respectively the identity matrix of size $n$ and null matrix of size $m \times n$. If the context allows it the dimensions of these matrices are often omitted. For a given matrix $B \in \mathrm{R}^{m \times n}$ such that $\operatorname{rank}(B)=r$, we define $B^{\perp} \in \mathrm{R}^{n \times(n-r)}$ the right orthogonal complement of $B$ by $B B^{\perp}=0$ and $B^{\perp} B^{\perp T}>0$. The set of complex numbers of the right-half of the complex plane is denoted $\mathrm{C}^{+}=\left\{s \in \mathrm{C}: s+s^{*} \geq \mathbf{0}\right\}$. The notation $\langle A\rangle$ stands for the symmetric matrix $A+A^{T}$. For block-diagonal matrices the following notation is adopted

$$
\operatorname{diag}\left(\begin{array}{c}
A \\
B \\
C
\end{array}\right)=\left[\begin{array}{ccc}
A & \mathbf{0} & \mathbf{0} \\
\mathbf{0} & B & \mathbf{0} \\
\mathbf{0} & \mathbf{0} & C
\end{array}\right]
$$

\subsection{Review of quadratic separation}

Well-posedness of feedback systems provides a fertile framework for stability analysis of non-linear and uncertain systems. Major results for robust stability analysis has been given in [8] and references therein. The purpose of this section is to briefly recall some new tools on quadratic separation developed for robustness issues of descriptor systems [15], which is needed for the main theorem of this paper.

Consider two possibly non-square matrices $\mathcal{E}$ and $\mathcal{A}$ and an uncertain constant, complex valued, matrix $\nabla$ with appropriate dimensions that belongs to some set $\nabla$. For simplicity of notations we assume in the present paper that $\mathcal{E}$ is full column rank. We make no assumption on the uncertainty set $\nabla$. 


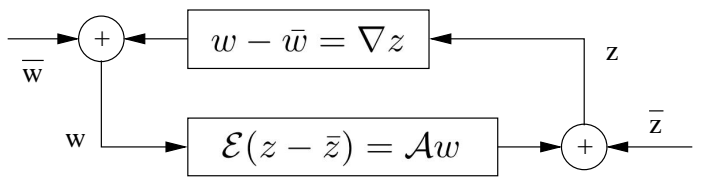

Figure 1: Feedback system

Theorem 1 [15] The uncertain feedback system of Figure 1 is well-posed if and only if there exists a Hermitian matrix $\Theta=\Theta^{*}$ satisfying both conditions

$$
\begin{gathered}
{\left[\begin{array}{cc}
\mathcal{E} & -\mathcal{A}
\end{array}\right]^{\perp *} \Theta\left[\begin{array}{ll}
\mathcal{E} & -\mathcal{A}
\end{array}\right]^{\perp}>\mathbf{0}} \\
{\left[\begin{array}{cc}
1 & \nabla^{*}
\end{array}\right] \Theta\left[\begin{array}{c}
\mathbf{1} \\
\nabla
\end{array}\right] \leq \mathbf{0}, \quad \forall \nabla \in \nabla .}
\end{gathered}
$$

If $\mathcal{E}$ and $\mathcal{A}$ are real matrices, the equivalence still holds with $\Theta$ restricted to be a real matrix.

A simple corollary of that result is for LTI system stability analysis. The system $\dot{x}(t)=A x(t)$, where $x(t) \in \mathrm{R}^{n}$, is stable if $A$ has all its eigenvalues in the left half plane, which is equivalent to the well-posedness of the feedback system of Figure 1 with $\mathcal{E}=\mathbf{1}_{n}, \mathcal{A}=A$ and $\boldsymbol{\nabla}=\left\{s^{-1} \mathbf{1}_{n}, s^{-1} \in \mathrm{C}^{+}\right\}$.

Another corollary is for delay-independent stability analysis of time-delay systems described by the equation

$$
\dot{x}(t)=A x(t)+A_{d} x(t-h) .
$$

Stability of that system whatever $h \geq 0$, can be recast as the well-posedness of the feedback system of Figure 1 with

$$
\boldsymbol{\nabla}=\left\{\left[\begin{array}{cc}
s^{-1} \mathbf{1}_{n} & \mathbf{0} \\
\mathbf{0} & e^{-s h} \mathbf{1}_{n}
\end{array}\right], s^{-1} \in \mathrm{C}^{+}, h \in \mathrm{R}^{+}\right\}
$$

and appropriate $\mathcal{E}$ and $\mathcal{A}$ matrices. Such LMI results can be found in [7] (and references therein) and are known to be conservative due to the lossly choice of separators $\Theta$.

The goal of the current paper is to extend these results for reducing conservatism and proving robust stability of time-delay systems with unknown delays in an interval. 


\section{Approximating the time-delay operator}

\subsection{Taylor series and Lagrange remainder}

Let $s \in \mathrm{C}$ and consider the function $\tau \rightarrow e^{-s \tau}$. Its Taylor series about the point $\tau_{0}=0$ writes as

$$
e^{-s \tau}=\sum_{i=0}^{k-1} \frac{1}{i !}(-s \tau)^{i}+R_{k}(s, \tau)
$$

where $R_{k}(s, \tau)$ is the Lagrange remainder. For any continuous time signal $v(t)$ recall that the $e^{-s \tau}$ and $s^{i}$ operators are such that

$$
e^{-s \tau}[v(t)]=v(t-\tau), s^{i}[v(t)]=v^{(i)}(t) .
$$

Define as well the operator $\delta_{i}(s, \tau)=i !(-s \tau)^{-i} R_{i}(s, \tau)$ and the $r_{i}(t)$ signal such that

$$
i !(-\tau)^{-i} R_{i}(s, \tau)[v(t)]=\delta_{i}(s, \tau)\left[v^{(i)}(t)\right]=r_{i}(t) .
$$

The Taylor series expansion imply for all $i \geq 0$ the following formulas relating $v(t-\tau)$ and the $v^{(i)}(t), r_{i}(t)$ signals:

$$
\begin{gathered}
v(t)=v(t-\tau)+\tau r_{1}(t) \\
v^{(i)}(t)=r_{i}(t)+\frac{\tau}{i+1} r_{i+1}(t)
\end{gathered}
$$

which indicates that in order to take advantage of the Taylor series expansion of the delay operator one needs:

- To artificially augment the system model with higher derivatives of the state. The notations involved in this model augmentation are given in the following section.

- To have some knowledge on the remainder representative $\delta_{i}(s, \tau)$. The information to be used in the following is of norm-bounded type. But at the difference of [6] a thinner bounded approximation is considered.

For $i=0$ one has $\delta_{0}(s, \tau)=e^{-s \tau}$ which sweeps the whole unit circle for $s \in \mathrm{C}^{+}$ but for $i \geq 1$ the domain in which the $\delta_{i}(s, \tau)$ operators lies is reduced and not centered at zero. For $i=1$ and $i=2$ these regions (their border is obtained for $s=j \omega$ ) are plotted on Figure 2. The circle centered at $c_{1}=0.249$ with radius $\alpha_{1}=0.751$ includes the region where lies $\delta_{1}(s, \tau)$ as proved in [22].

Lemma 1 If for all $s \in \mathrm{C}^{+}$and all $\theta \in[0 \tau]$ the complex number $\delta_{i}(s, \theta)$ belongs to the disc centered at $c_{i}$ with radius $\alpha_{i}$, then the same property holds for $\delta_{j}(s, \theta)$ with $j \geq i$.

Proof: Assume $\delta_{i}(s, \theta)$ is in the disc centered at $c_{i}$ with radius $\alpha_{i}$ :

$$
\alpha_{i}^{-1}\left(\delta_{i}(s, \theta)-c_{i}\right)^{2} \leq \alpha_{i} .
$$




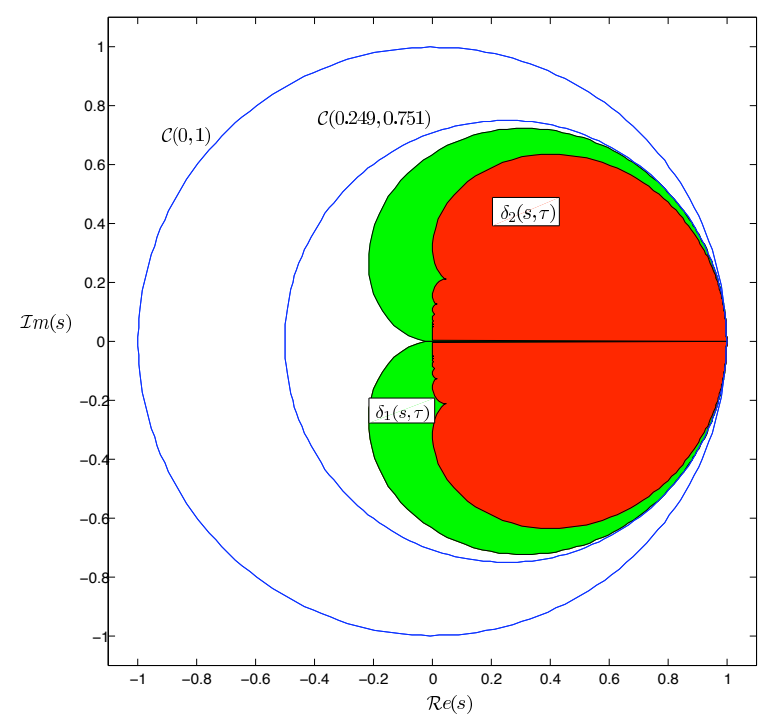

Figure 2: Feedback system

Apply a Schur complement argument to get

$$
\left[\begin{array}{cc}
\alpha_{i} & \delta_{i}(s, \theta)-c_{i} \\
\delta_{i}(s, \theta)-c_{i} & \alpha_{i}
\end{array}\right] \leq \mathbf{0}
$$

which also writes as $\Lambda_{i}(s, \theta) \geq \mathbf{0}$ where:

$$
\Lambda_{i}(s, \theta)=\left[\begin{array}{cc}
\alpha_{i} \theta^{i} / i ! & (-s)^{-i} R_{i}(s, \theta)-c_{i} \\
(-s)^{-i} R_{i}(s, \theta)-c_{i} & \alpha_{i} \theta^{i} / i !
\end{array}\right] .
$$

Note that

$$
\begin{aligned}
\int_{0}^{\hat{\theta}} R_{i}(s, \theta) d \theta=\int_{0}^{\hat{\theta}} e^{-s \tau} & -\sum_{i=0}^{k-1} \frac{1}{i !}(-s \tau)^{i} d \theta \\
= & (-s)^{-1} R_{i+1}(s, \hat{\theta})
\end{aligned}
$$

hence taking the integral of $\Lambda_{i}(s, \theta)$ gives for any $\hat{\theta} \in[0 \tau]$

$$
\mathbf{0} \leq \int_{0}^{\hat{\theta}} \Lambda_{i}(s, \theta) d \theta=\Lambda_{i+1}(s, \hat{\theta})
$$

which with the converse Schur complement argument implies that $\delta_{i+1}(s, \hat{\theta})$ belongs to the disc centered at $c_{i}$ with radius $\alpha_{i}$. By induction the property holds for all $j \geq i$.

Definition 1 Two vectors $c=\left(\begin{array}{lll}c_{1} & \ldots & c_{k}\end{array}\right)$ and $\alpha=\left(\begin{array}{ccc}\alpha_{1} & \ldots & \alpha_{k}\end{array}\right)$ are said to be Taylor-remainder valid if $\left|\delta_{i}(s, \tau)-c_{i}\right| \leq \alpha_{i}$ for all $s \in \mathrm{C}^{+}$and all $i=1 \ldots k$. 
In the numerical examples treated in the last section the vectors are such that $c_{i}=$ 0.249 and $\alpha_{i}=0.751$. They are Taylor-remainder valid due to lemma 1 .

\subsection{Fractioning the delay}

The quality of the truncated Taylor series at order $k$ depends on the approximation of the $\delta_{k}(s, \tau)$ representative of the remainder which is exposed upper. Yet another property of Taylor series is that the truncation is less and less conservative as $\tau$ tends to zero. For this purpose, assuming a delay $h$, the methodology is applied to a fraction of the delay $\tau=h / q$. As in [5], based on the property that

$$
\begin{aligned}
v(t-h) & =e^{-s h / q}\left[v\left(t-\frac{(q-1) h}{q}\right)\right] \\
& =e^{-s 2 h / q}\left[v\left(t-\frac{(q-2) h}{q}\right)\right]=\cdots=e^{-s h}[v(t)],
\end{aligned}
$$

the fractioning implies to augment the system model with all $v\left(t-\frac{l h}{q}\right)$ signals were $l=0 \ldots q$. [4] demonstrates that these augmented signals allow implicitly to prove stability with Lyapunov functions involving the $q$ samples $v\left(t-\frac{l h}{q}\right)$ of the signal $v(\theta)$ for $\theta \in[t-h t]$. The whole state of a time-delay system defined as $x(\theta), \theta \in[t-h t]$ can hence be taken into account asymptotically as $q$ goes to infinity.

\section{Main result}

\subsection{Augmented model}

Let the following time-delay system with one delay $h$ assumed constant:

$$
\left\{\begin{array}{l}
\dot{x}(t)=A x(t)+A_{d} x(t-h), \forall t \geq 0 \\
x(t)=\phi(t), \forall t \in[-h, 0]
\end{array}\right.
$$

where $x(t) \in \mathrm{R}^{n}$ is the instantaneous state and $\phi$ is the initial condition. To reduce numerical complexity of the results in case $A_{d}$ is not full rank, let any factorization $A_{d}=B C$. The effectively delayed part of the state is denoted $v(t-h)=C x(t-h)$ with $v \in \mathrm{R}^{p}$ :

$$
\left\{\begin{array}{l}
\dot{x}(t)=A x(t)+B v(t-h) \\
v(t)=C x(t)
\end{array}\right.
$$

The augmented model with Taylor series stopped at degree $k$ with delay fractioning $q$ which includes all possible relations described above is now defined. First, let the vectors of all derivatives up to order $k-1$ :

$$
\tilde{x}(t)=\left(\begin{array}{c}
x^{(k-1)}(t) \\
\vdots \\
\dot{x}(t) \\
x(t)
\end{array}\right), \tilde{v}(t)=\left(\begin{array}{c}
v^{(k-1)}(t) \\
\vdots \\
\dot{v}(t) \\
v(t)
\end{array}\right) .
$$


Second, let the vector of delayed signals for all delays of the fractioning:

$$
\hat{v}(t)=\left(\begin{array}{c}
\tilde{v}\left(t-\frac{q-1}{q} h\right) \\
\vdots \\
\tilde{v}\left(t-\frac{1}{q} h\right) \\
\tilde{v}(t)
\end{array}\right) .
$$

Finally, let the vectors of remainder signals with their derivatives and the vectors of signals on which apply the operators $\delta_{i}$ :

$$
\tilde{r}_{i}(t)=\left(\begin{array}{c}
r_{i}^{(k-i)}(t) \\
\vdots \\
\dot{r}_{i}(t) \\
r_{i}(t)
\end{array}\right), \tilde{v}_{i}(t)=\left(\begin{array}{c}
v_{i}^{(k)}(t) \\
\vdots \\
v_{i}^{(i+1)}(t) \\
v_{i}^{(i)}(t)
\end{array}\right) .
$$

The relationships between these vectors can all be formulated in terms of a feedback connected system of Figure 1. Choosing the vectors

$$
z=\left(\begin{array}{c}
\dot{\tilde{x}}(t) \\
\hat{v}(t) \\
\tilde{v}_{1}(t) \\
\vdots \\
\tilde{v}_{k}(t)
\end{array}\right), w=\left(\begin{array}{c}
\tilde{x}(t) \\
\hat{v}\left(t-\frac{h}{q}\right) \\
\tilde{r}_{1}(t) \\
\vdots \\
\tilde{r}_{k}(t)
\end{array}\right)
$$

the "uncertainty" that gathers all involved operators is defined as

$$
\nabla=\operatorname{diag}\left(\begin{array}{c}
s^{-1} \mathbf{1}_{n k} \\
\delta_{0}\left(s, \frac{h}{q}\right) \mathbf{1}_{k p q} \\
\delta_{1}\left(s, \frac{h}{q}\right) \mathbf{1}_{k p} \\
\vdots \\
\delta_{k}\left(s, \frac{h}{q}\right) \mathbf{1}_{p}
\end{array}\right)
$$

and the matrices $\mathcal{E}$ and $\mathcal{A}$ are constructed by the following equations:

- Augmented system equations (6)

$$
\begin{aligned}
& \dot{\tilde{x}}(t)=\left(\mathbf{1}_{k} \otimes A\right) \tilde{x}(t) \\
& +\left(\mathbf{1}_{k} \otimes B\right)\left[\begin{array}{ll}
\mathbf{1}_{k p} & \mathbf{0}_{k p, k p(q-1)}
\end{array}\right] \hat{v}\left(t-\frac{h}{q}\right) \\
& {\left[\begin{array}{ll}
\mathbf{0}_{k p, k p(q-1)} & \mathbf{1}_{k p}
\end{array}\right] \hat{v}(t)=\left(\mathbf{1}_{k} \otimes C\right) \tilde{x}(t)} \\
& \left(\mathbf{1}_{k} \otimes C\right) \dot{\tilde{x}}(t)-\tilde{v}_{1}(t)=0
\end{aligned}
$$


- Internal relationships between the signals:

$$
\begin{aligned}
& {\left[\begin{array}{ll}
\mathbf{0}_{(k-1) n, n} & \mathbf{1}_{(k-1) n}
\end{array}\right] \dot{\tilde{x}}(t)} \\
& =\left[\begin{array}{ll}
\mathbf{1}_{(k-1) n} & \mathbf{0}_{(k-1) n, n}
\end{array}\right] \tilde{x}(t) \\
& {\left[\begin{array}{ll}
\mathbf{1}_{k p(q-1)} & \mathbf{0}_{k p(q-1), k p}
\end{array}\right] \hat{v}(t)} \\
& =\left[\begin{array}{ll}
\mathbf{0}_{k p(q-1), k p} & \mathbf{1}_{k p(q-1)}
\end{array}\right] \hat{v}\left(t-\frac{h}{q}\right) \\
& {\left[\begin{array}{ll}
\mathbf{1}_{(k-i) p} & \mathbf{0}_{(k-i) p, k p}
\end{array}\right] \tilde{v}_{i}(t)-\tilde{v}_{i+1}(t)=0}
\end{aligned}
$$

- Equations obtained from the Taylor series formula (4)

$$
\begin{aligned}
& {\left[\begin{array}{ll}
\mathbf{0}_{k p, k p(q-1)} & \mathbf{1}_{k p}
\end{array}\right] \hat{v}(t)} \\
& \quad=\left[\begin{array}{ll}
\mathbf{0}_{k p, k p(q-1)} & \mathbf{1}_{k p}
\end{array}\right] \hat{v}\left(t-\frac{h}{q}\right)+\frac{h}{q} \tilde{r}_{1}(t) \\
& {\left[\begin{array}{lll}
\mathbf{0}_{(k-i) p, k p(q-1)} & \mathbf{1}_{(k-i) p} & \mathbf{0}_{(k-i) p, p i}
\end{array}\right] \hat{v}(t)} \\
& \quad=\left[\begin{array}{ll}
\mathbf{0}_{(k-i) p, p} & \mathbf{1}_{(k-i) p}
\end{array}\right] \tilde{r}_{i}(t)+\frac{h}{(i+1) q} \tilde{r}_{i+1}(t)
\end{aligned}
$$

The obtained matrix $\mathcal{E}$ is full rank.

\subsection{LMI conditions for stability analysis}

Based on the modeling described above, Theorem 1 can be applied.

Theorem 2 Given $k$ the upper degree of Taylor series, $q$ the delay fractioning and $(c, \alpha)$ a Taylor-remainder valid couple, let $\mathcal{L}(k, q, c, \alpha)$ be the LMI problem obtained applying Theorem 1 with $\Theta=$

$$
\left[\begin{array}{cr}
\operatorname{diag}\left(\begin{array}{c}
\mathbf{0} \\
-Q_{0} \\
\left(c_{1}^{2}-\alpha_{1}^{2}\right) Q_{1} \\
\vdots \\
\left(c_{k}^{2}-\alpha_{k}^{2}\right) Q_{k}
\end{array}\right) & \text { diag }\left(\begin{array}{c}
-P \\
\mathbf{0} \\
-c_{1} Q_{1} \\
\vdots \\
-c_{k} Q_{k}
\end{array}\right) \\
\operatorname{diag}\left(\begin{array}{c}
\mathbf{0} \\
Q_{0} \\
-c_{1} Q_{1} \\
\vdots \\
-c_{k} Q_{k}
\end{array}\right) & \operatorname{diag}\left(\begin{array}{c}
Q_{1} \\
\vdots \\
Q_{k}
\end{array}\right)
\end{array}\right]
$$

where the matrices $P, Q_{i}$ are all symmetric, $P$ is positive definite and the $Q_{i}$ are positive semi-definite. The time-delay system (5) is stable if $\mathcal{L}(k, q, c, \alpha)$ is feasible. 
Proof: Take $\Theta$ as (8) with $\nabla$ defined in (7):

$$
\left[\begin{array}{c}
\mathbf{1} \\
\nabla
\end{array}\right]^{*} \Theta\left[\begin{array}{c}
\mathbf{1} \\
\nabla
\end{array}\right]=\operatorname{diag}\left(\begin{array}{c}
-\left(s+s^{*}\right) P \\
\left(e^{-2 s h / q}-1\right) Q_{0} \\
\left(\left|\delta_{1}\left(s, \frac{h}{q}\right)-c_{1}\right|^{2}-\alpha_{1}^{2}\right) Q_{1} \\
\vdots \\
\left(\left|\delta_{k}\left(s, \frac{h}{q}\right)-c_{k}\right|^{2}-\alpha_{k}^{2}\right) Q_{k}
\end{array}\right)
$$

which proves (2) for $s \in \mathrm{C}^{+}$.

Note that stability proved by Theorem 2 corresponds implicitly to some Lyapunov function $V(x)$ such that

$$
\dot{V}(x)=-\left(\begin{array}{cc}
z^{*} & w^{*}
\end{array}\right) \Theta\left(\begin{array}{c}
z \\
w
\end{array}\right) .
$$

It depends both on the $k$ first derivatives of the state and on the delayed state evaluated for the $q$ fractions of the interval $[t-h t]$.

Numerically, the LMI problem $\mathcal{L}(k, q, c, \alpha)$ has the following characteristics:

- The constraint (1) has $n+k p q$ rows and columns. The additional constraints $P>\mathbf{0}, Q_{0} \geq \mathbf{0}$ and $Q_{i} \geq \mathbf{0}$ have respectively $n k, k p q$ and $(k-i+1) p$ rows and columns. The overall size of the LMIs is

$$
(k+1) n+(2 q+(k+1) / 2) k p .
$$

- The decision variables are the symmetric matrices of $\Theta$. The number of scalar decision variables is $k n(k n+1) / 2$ for $P, k p q(k p q+1) / 2$ for $Q_{0}$ and $(k-i+$ $1)(k-i+2) / 2$ for the $Q_{i}$ matrices. The overall number of decision variables is

$$
\frac{k n}{2}(k n+1)+\frac{k p q}{2}(k p q+1)+\frac{k p}{6}\left(2 k^{2} p+3 k(p+1)+p+3\right) .
$$

These comments on the dimensions of the LMI problem $\mathcal{L}(k, q, c, \alpha)$ clearly indicate that even if one can prove some asymptotic property as both $k$ and $q$ grow to infinity, it would be numerically intractable. Still some properties with respect to conservatism reduction can be produced.

\subsection{Conservatism reduction}

Proposition 1 Let two couple of vectors $(c, \alpha)$ and $(\hat{c}, \hat{\alpha})$ such that, for all $i$, the disc centered at $\hat{c}_{i}$ with radius $\hat{\alpha}_{i}$ is included in the disc centered at $c_{i}$ with radius $\alpha_{i}$. In such a case, if $\mathcal{L}(k, q, c, \alpha)$ is feasible, $\mathcal{L}(k, q, \hat{c}, \hat{\alpha})$ is feasible as well.

Proof: $\delta_{i}$ lies in the circle centered at $c_{i}$ with radius $\alpha_{i}$ is and only if the following quadratic constraints holds

$$
\left(\begin{array}{ll}
1 & \delta_{i}
\end{array}\right)\left[\begin{array}{cc}
c_{i}^{2}-\alpha_{i}^{2} & -c_{i} \\
-c_{i} & 1
\end{array}\right]\left(\begin{array}{c}
1 \\
\delta_{i}
\end{array}\right) \leq \mathbf{0} .
$$


S-procedure indicates that the conditions assumed on $(c, \alpha)$ and $(\hat{c}, \hat{\alpha})$ imply that there exists a positive scalar $\epsilon_{i}$ such that

$$
\left[\begin{array}{cc}
\hat{c}_{i}^{2}-\hat{\alpha}_{i}^{2} & -\hat{c}_{i} \\
-\hat{c}_{i} & 1
\end{array}\right] \geq \epsilon_{i}\left[\begin{array}{cc}
c_{i}^{2}-\alpha_{i}^{2} & -c_{i} \\
-c_{i} & 1
\end{array}\right]
$$

which suffices to conclude taking $\hat{\Theta}(\hat{c}, \hat{\alpha})$ defined by $\hat{P}=P, \hat{Q}_{0}=Q_{0}$ and $\hat{Q}_{i}=\epsilon_{i} Q_{i}$ for all $i=1 \ldots k$.

Proposition 1 indicates that by improving the approximation of the $\delta_{i}$ operators, the LMI conditions become less conservative. The results exposed here are therefore less conservative than those of [6] in which all the $\delta_{i}$ operators were restricted to lie in the unit circle centered at zero.

Proposition 2 If $\mathcal{L}(k, q, c, \alpha)$ is feasible then for all larger degrees of the Taylor series $\hat{k} \geq k, \mathcal{L}(\hat{k}, q, c, \alpha)$ is feasible as well.

The proof is identical to that in [6] and is therefore omitted for space reasons. The sketch of the proof is starting from a separator for degree $k$ to build the separator for degree $\hat{k}=k+1$ of the type (8) with variables build with structure

$$
\hat{P}=\left[\begin{array}{cc}
\epsilon_{P} \mathbf{1} & \mathbf{0} \\
\mathbf{0} & P
\end{array}\right], \hat{Q}_{i}=\left[\begin{array}{cc}
\epsilon_{i} \mathbf{1} & \mathbf{0} \\
\mathbf{0} & Q_{i}
\end{array}\right]
$$

where $\epsilon_{P}$ and $\epsilon_{i}$ are small enough positive scalars.

Proposition 3 If $\mathcal{L}(k, q, c, \alpha)$ is feasible then for any thinner fractioning $\hat{q} \geq q, \mathcal{L}(k, \hat{q}, c, \alpha)$ is feasible as well.

Proof: Let $M$ be the $\left[\begin{array}{ll}\mathcal{E} & -\mathcal{A}\end{array}\right]$ matrix for a fractioning $q$ and $\hat{M}$ be the same matrix for fractioning $\hat{q}=q+1$. Let $\theta$ be a solution to the LMI problem $\mathcal{L}(k, q, c, \alpha)$. Due to inverse elimination lemma [17] applied to (1), there exists a matrix $Y$ such that

$$
\Theta>Y M+M^{T} Y^{T} .
$$

With properly arranged rows, $M$ can be partitioned as

$$
M=\left[\begin{array}{ccccc}
\mathbf{0} & \mathbf{1}_{k p(q-1)} & \mathbf{0} & -\mathbf{1}_{k p(q-1)} & \mathbf{0} \\
N_{1} & \mathbf{0} & N_{2} & N_{3} & N_{4}
\end{array}\right]
$$

and according to this partitioning define

$$
Y^{T}=\left[\begin{array}{lllll}
Y_{11} & Y_{12} & Y_{13} & Y_{14} & Y_{15} \\
Y_{21} & Y_{22} & Y_{23} & Y_{24} & Y_{25}
\end{array}\right]
$$

Let as well the partitioning

$$
Q_{0}=\left[\begin{array}{ll}
Q_{01} & Q_{02} \\
Q_{02}^{T} & Q_{03}
\end{array}\right], \quad \begin{aligned}
& Q_{01} \in \mathrm{R}^{k p \times k p} \\
& Q_{03} \in \mathrm{R}^{k p(q-1) \times k p(q-1)} .
\end{aligned}
$$


For the fractioning $\hat{q}=q+1, \hat{M}$ is exactly

$$
\left[\begin{array}{ccccccc}
\mathbf{0} & \mathbf{1}_{k p} & \mathbf{0} & \mathbf{0} & -\mathbf{1}_{k p} & \mathbf{0} & \mathbf{0} \\
\mathbf{0} & \mathbf{0} & 1_{k p(q-1)} & \mathbf{0} & \mathbf{0} & -\mathbf{1}_{k p(q-1)} & \mathbf{0} \\
N_{1} & \mathbf{0} & \mathbf{0} & N_{2} & \mathbf{0} & N_{3} & N_{4}
\end{array}\right] .
$$

Let the matrix $\hat{Y}^{T}=$

$$
\left[\begin{array}{ccccccc}
\mathbf{0} & -\frac{1}{2} Q_{01} & -\frac{1}{2} Q_{02} & \mathbf{0} & -\frac{1}{2} Q_{01} & -\frac{1}{2} Q_{02} & \mathbf{0} \\
Y_{11} & -\frac{1}{2} Q_{02}^{T} & Y_{12} & Y_{13} & -\frac{1}{2} Q_{02}^{T} & Y_{14} & Y_{15} \\
Y_{21} & \mathbf{0} & Y_{22} & Y_{23} & \mathbf{0} & Y_{24} & Y_{25}
\end{array}\right]
$$

and let $\hat{\Theta}$ be such as (8) with matrices $\hat{P}=P$ and $\hat{Q}_{i}=Q_{i}$ equal to those of $\Theta$ for all $i=1 \ldots k$, and with

$$
\hat{Q}_{0}=\left[\begin{array}{ccc}
Q_{01} & \mathbf{0} & Q_{02} \\
\mathbf{0} & Q_{01} & Q_{02} \\
Q_{02}^{T} & Q_{02}^{T} & Q_{03}
\end{array}\right]
$$

The matrices defined in this way happen to satisfy

$$
\hat{\Theta} \geq \hat{Y} \hat{M}+\hat{M}^{T} \hat{Y}^{T}
$$

compared with the inequality for fractioning $q$ it simply corresponds to insertion of zero rows and columns. Inverse elimination lemma and a small perturbation argument gives (1) for $\hat{q}=q+1$. The result extends to any $\hat{q} \geq q$ by induction.

\subsection{Some important sub-cases}

One major sub-case of time-delay systems is when stability is independent of the value of the delay (IOD-stability). The apparently delay-dependent LMI formulations given above do allow to handle this case.

Proposition 4 If $\mathcal{L}(k, q, c, \alpha)$ is feasible with a $\Theta$ matrix restricted to have $Q_{i}=\mathbf{0}$ for all $i=1 \ldots k$, then the system is stable whatever the value of the delay $h$.

This result is based on the fact that the LMI conditions become delay-independent when $Q_{i}=\mathbf{0}$ for $i=1 \ldots k$. A particularity is that the fractioning does not reduce conservatism in such a case: one can restrict to $q=1$. Aside from that, introducing higher order derivatives by increasing $k$ does reduce conservatism. The problem $\mathcal{L}(k, 1, c, \alpha)$ with $Q_{i}=\mathbf{0}$ for all $i=1 \ldots k$ happens to be exactly that of [2]. Note as well that $\mathcal{L}(0, q, c, \alpha)$ can be feasible only for IOD-stable systems. If stability depends of the delay, the LMI conditions may be feasible only if $k \geq 1$.

A second major sub-case is known as "delay-dependent" stability (DD-stability). It corresponds to systems stable for zero delay, the problem being to find the maximal delay $\bar{h}$ such that the system is stable for all $0 \leq h \leq \bar{h}$. 
Proposition 5 If $\mathcal{L}(k=1, q, c, \alpha)$ is feasible for a delay $\bar{h}$, then the system is stable whatever $h \in[0 \bar{h}]$.

This result is demonstrated in [5]. It also shows that if the system is not stable for $h=0$, then $\mathcal{L}(k, q, c, \alpha)$ cannot be feasible unless $k \geq 2$. Only for $k \geq 2$ the LMI conditions can prove interval-delay stability.

\section{Robustness issues}

As for most analysis problems formulated in terms of LMIs, the results of the previous section may be extended including robustness issues. In the current paper, affine polytopic uncertain models with interval time-delays are considered:

$$
\left\{\begin{array}{l}
\dot{x}(t)=A(\xi) x(t)+B(\xi) v(t-h) \\
v(t)=C(\xi) x(t)
\end{array}\right.
$$

with $h \in[\underline{h} \bar{h}]$ and

$$
\left[\begin{array}{cc}
A(\xi) & B(\xi) \\
C(\xi) & \mathbf{0}
\end{array}\right]=\sum_{i=1}^{N} \xi_{i}\left[\begin{array}{cc}
A^{[i]} & B^{[i]} \\
C^{[i]} & \mathbf{0}
\end{array}\right]
$$

where $\xi=\left(\begin{array}{lll}\xi_{1} & \ldots & \xi_{N}\end{array}\right)$ belongs to the set $\Xi=\left\{\xi_{i} \geq \mathbf{0}, \quad \sum_{i=1}^{N} \xi_{i}=1\right\}$. Note that the $\mathcal{E}$ and $\mathcal{A}$ matrices are linear with respect to both the model parameters $A, B$ and $C$ and with respect to the delay $h$. More precisely, denote $M(\xi, h)$ the parameter dependent $\left[\begin{array}{ll}\mathcal{E} & -\mathcal{A}\end{array}\right]$ matrix. It can decomposed as

$$
M(\xi, h)=M_{0}+M(\xi)+h M_{d}=M_{0}+\sum_{i=1}^{N} \xi_{i} M^{[i]}+h M_{d} .
$$

Theorem 3 If there exist $2 N$ separators $\left(\underline{\Theta}^{[i]}, \bar{\Theta}^{[i]}\right)$ structured as (8) and a matrix $Y$ such that the following LMIs hold for all $i=1 \ldots N$

$$
\begin{aligned}
& \underline{\Theta}^{[i]}>\left\langle Y\left(M_{0}+M^{[i]}+\underline{h} M_{d}\right)\right\rangle \\
& \bar{\Theta}^{[i]}>\left\langle Y\left(M_{0}+M^{[i]}+\bar{h} M_{d}\right)\right\rangle
\end{aligned}
$$

then the time delay system (9) is robustly stable.

Proof: Define the parameter-dependent separator

$$
\Theta(\xi, h)=\sum_{i=1}^{N} \xi_{i}\left(\frac{\underline{h}-h}{\underline{h}-\bar{h}} \bar{\Theta}^{[i]}+\frac{\bar{h}-h}{\bar{h}-\underline{h}^{\underline{\Theta}}}{ }^{[i]}\right)
$$


The inequalities (10) are all linear with respect to $\underline{\Theta}^{[i]}, \bar{\Theta}^{[i]}, M^{[i]}, \underline{h}$ and $\bar{h}$ therefore taking linear combinations of these inequalities implies that

$$
\Theta(\xi, h)>\left\langle Y\left(M_{0}+M(\xi)+h M_{d}\right)\right\rangle=\langle Y M(\xi, h)\rangle
$$

holds for all $\xi \in \Xi$ and all $h \in[\underline{h} \bar{h}]$. Post and pre multiply the last inequality by $M^{\perp}(\xi, h)$ and its transpose respectively to get (1) which concludes the proof.

\section{Numerical examples}

The latest version of SeDuMi [18] (SeDuMi 1.1 available at http: // sedumi.mcmaster.ca/) is used to solve the LMIs along with the parser YALMIP [12]. Computation is done on a Linux PC computer with i686 processor and 2GB memory.

\subsection{First example}

Theorem 3 is applied to the academic example $\ddot{y}(t)-0.1 \dot{y}(t)+2 y(t)=y(t-h)$ taken from [7] for which analytical analysis indicates ] 0.100168261 .7178 [ is the actual stabilizing open interval. Only $h$ is assumed uncertain. It is chosen to lie in the interval $[\underline{h} \bar{h}]$ with $\underline{h}=0.101$ fixed. The goal is by dichotomy to find the maximal $\bar{h}$. Results for various values of $k$ and $q$ are given in Table 1 . The LMIs fail for $k<2$ as expected (see section IV-D). Tests are limited to problems with less than 160 decision variables. For this example conservatism reduces faster for growing values of fractioning $q$ than with respect to Taylor series degree $k$.

The number of variables of each LMI problem is given in Table 2. The computation time is less than 2 minutes for all individual LMI problems.

Table 1: $\bar{h}$ obtained by Theorem 3 for first example

\begin{tabular}{|c|c|c|c|c|c|}
\hline & $\mathrm{q}=1$ & $\mathrm{q}=2$ & $\mathrm{q}=3$ & $\mathrm{q}=4$ & $\mathrm{q}=5$ \\
\hline$k=2$ & 0.7062 & 1.6997 & 1.7137 & 1.7163 & 1.7171 \\
$k=3$ & 1.5689 & 1.7026 & 1.7147 & 1.7166 & 1.7172 \\
$k=4$ & 1.5689 & 1.7038 & & & \\
\hline
\end{tabular}

To evaluate the conservatism of Theorem 3 compared to Theorem 2, a dichotomy line search is performed for $k=3$ and $q=5$ to find the maximal value of $h$ that makes $\mathcal{L}(k, q, c, \alpha)$ feasible: $\bar{h}=1.7174$. As expected it is better than the robust values obtained with Theorem 3, but it proves nothing for the interval [ 0.1011 .7174$]$. 
Table 2: Number of decision variables (Theorem 3)

\begin{tabular}{|c|c|c|c|c|c|}
\hline & $\mathrm{q}=1$ & $\mathrm{q}=2$ & $\mathrm{q}=3$ & $\mathrm{q}=4$ & $\mathrm{q}=5$ \\
\hline$k=2$ & 55 & 67 & 79 & 91 & 103 \\
$k=3$ & 91 & 109 & 127 & 145 & 163 \\
$k=4$ & 133 & 157 & & & \\
\hline
\end{tabular}

\subsection{Second example}

The DD-stable system of [22] is tested for $K=1$. The same procedure as above is adopted with $\underline{h}=0$. Results are reported in Table 3. For this example increasing the Taylor series degree has a major contribution for getting close to the actual maximal bound 1.4247 .

Table 3: $\bar{h}$ obtained by Theorem 3 for second example

\begin{tabular}{|c|c|c|c|c|c|}
\hline & $\mathrm{q}=1$ & $\mathrm{q}=2$ & $\mathrm{q}=3$ & $\mathrm{q}=4$ & $\mathrm{q}=5$ \\
\hline$k=1$ & 0.2518 & 0.2531 & 0.2533 & 0.2534 & 0.2535 \\
$k=2$ & 1.0570 & 1.2946 & 1.3340 & 1.3454 & 1.3496 \\
$k=3$ & 1.2325 & 1.3997 & 1.4190 & & \\
\hline
\end{tabular}

The interesting feature of Theorem 3 is that stability is guaranteed for all the interval $[0 \bar{h}]$. If removing this robustness specification and searching by dichotomy the maximal stabilizing delay with the help of Theorem 2 gives even better bounds (higher values of $k$ can be tested because numerical complexity is about 10 times lower). Tests are done for various values of $k=q$ and for two choices of the $(c, \alpha)$ Taylor-remainder valid vectors. Results are given in Table 4 . As expected due to Proposition 1 proper approximation of the regions where lie the $\delta_{i}(s, \tau)$ operators has a non negligible effect on conservatism. This effect seems to vanish for large values of $k$ and $q$ but this is at the expense of increasing the numerical burden.

Table 4: $\bar{h}$ obtained by Theorem 2 for second example

\begin{tabular}{|l|l|l|l|l|l|}
\hline & $\mathrm{k}=\mathrm{q}=1$ & $\mathrm{k}=\mathrm{q}=2$ & $\mathrm{k}=\mathrm{q}=3$ & $\mathrm{k}=\mathrm{q}=4$ & $\mathrm{k}=\mathrm{q}=5$ \\
\hline$\left(c_{i}, \alpha_{i}\right)=(0,1)$ & 0.1268 & 1.1644 & 1.3844 & 1.4019 & 1.4236 \\
$\left(c_{i}, \alpha_{i}\right)=$ & & & & 1.4233 & 1.4243 \\
$(0.249,0.751)$ & 0.2518 & 1.2947 & 1.4197 & 1.423 \\
\hline
\end{tabular}




\section{Conclusions}

A novel method for stability analysis of time-delay systems is provided in the quadratic separation framework. Inherent conservatism of LMI-based methods is reduced by the means of three techniques. One is fractioning of the delay. The second is Taylor series representation of the delay operator. The third is norm-bounded representation of the Taylor series remainders in circles not centered at zero. This last representation may be further improved as illustrated in Figure 2: there is a wide gap between the $\delta_{2}(s, \tau)$ region and the circle we used. Further developments in this direction will be proposed in the close future.

\section{References}

[1] A. M. Annaswamy, M. Fleifil, J. P. Hathout, and A. F. Ghoniem. Impact of linear coupling on the design of active controllers for thermoacoustic instability. Combust. Sci. Technol., 128:131-160, 1997.

[2] P.-A. Bliman. LMI characterization of the strong delay-independent stability of linear delay systems via quadratic Lyapunov-krasovskii functionals. Systems \& Control Letters, 43:263-274, 2001.

[3] P.-A. Bliman and T. Iwasaki. LMI characterisation of robust stability for timedelay systems: singular perturbation approach. In IEEE Conference on Decision and Control, San Diego, December 2006.

[4] F. Gouaisbaut and D. Peaucelle. Delay-dependent robust stability of time delay systems. In IFAC Symposium on Robust Control Design, Toulouse, July 2006. Paper in an invited session.

[5] F. Gouaisbaut and D. Peaucelle. Delay-dependent stability analysis of linear time delay systems. In IFAC Workshop on Time Delay Systems, L'Aquila, Italy, July 2006.

[6] F. Gouaisbaut and D. Peaucelle. Stability of time-delay systems with non-small delay. In IEEE Conference on Decision and Control, San Diego, December 2006.

[7] K. Gu, K.L. Kharitonov, and J. Chen. Stability of Time-Delay Systems. Control Engineering Series. Birkhauser, Boston USA, 2003.

[8] T. Iwasaki and S. Hara. Well-posedness of feedback systems: Insights into exact robustness analysis and approximate computations. IEEE Trans. on Automat. Control, 43(5):619-630, 1998.

[9] S. Jayaram, S. G. Kapoor, and R. E. DeVor. Analytical stability analysis of variable spindle speed machines. J. Manufact. Eng., 122:391-397, 2000. 
[10] C.R. Knospe and M. Roozbehani. Stability of linear systems with interval time delays excluding zero. IEEE Transactions on Automatic Control, 51(8):12711288, August 2006.

[11] V.B. Kolmanovskii and A. Myshkis. Introduction to the theory and applications of functional differential equations. Kluwer Acad., Dordrecht, 1999.

[12] J. Löfberg. YALMIP : A Toolbox for Modeling and Optimization in MATLAB, 2004.

[13] N. Olgac and R. Sipahi. An exact method for the stability analysis of time delayed LTI systems. IEEE Transactions on Automatic Control, 47(5):793?797, May 2002.

[14] N. Olgac and R. Sipahi. Direct method for analyzing the stability of neutral type lti-time delayed systems. Automatica, 40(5):847?853, May 2004.

[15] D. Peaucelle, D. Arzelier, D. Henrion, and F. Gouaisbaut. Quadratic separation for feedback connection of an uncertain matrix and an implicit linear transformation. Automatica, 2007. doi: 10.1016/j.automatica.2006.11.005.

[16] J.-P. Richard. Time delay systems: An overview of some recent advances and open problems. Automatica, 39(10):1667-1694, 2003.

[17] R.E. Skelton, T. Iwasaki, and K. Grigoriadis. A unified Approach to Linear Control Design. Taylor and Francis series in Systems and Control, 1998.

[18] J.F. Sturm. Using SeDuMi 1.02, a MATLAB toolbox for optimization over symmetric cones. Optimization Methods and Software, 11-12:625-653, 1999. URL: fewcal.kub.nl / sturm/software/sedumi.html.

[19] V. Suplin, E. Fridman, and U. Shaked. $h_{i} n f t y$ control of linear uncertain timedelay systemsa projection approach. IEEE Trans. Aut. Control, 51(4):680- 685, 2006.

[20] J. Zhang, C.R. Knopse, and P. Tsiotras. Stability of time-delay systems: Equivalence between Lyapunov and scaled small-gain conditions. IEEE Trans. on Automat. Control, 46(3):482-486, March 2001.

[21] J. Zhang, C.R. Knospe, and Tsiotras. New results for the analysis of linear systems with time invariant delays. International Journal of Robust and Nonlinear Control, 13:1149-1175, 2003.

[22] J. Zhang, C.R. Knospe, and P. Tsiotras. Toward less conservative stability analysis of time delay systems. In IEEE Conference on Decision and Control, pages 20172022, Phoenix, Arizona, December 1999. 